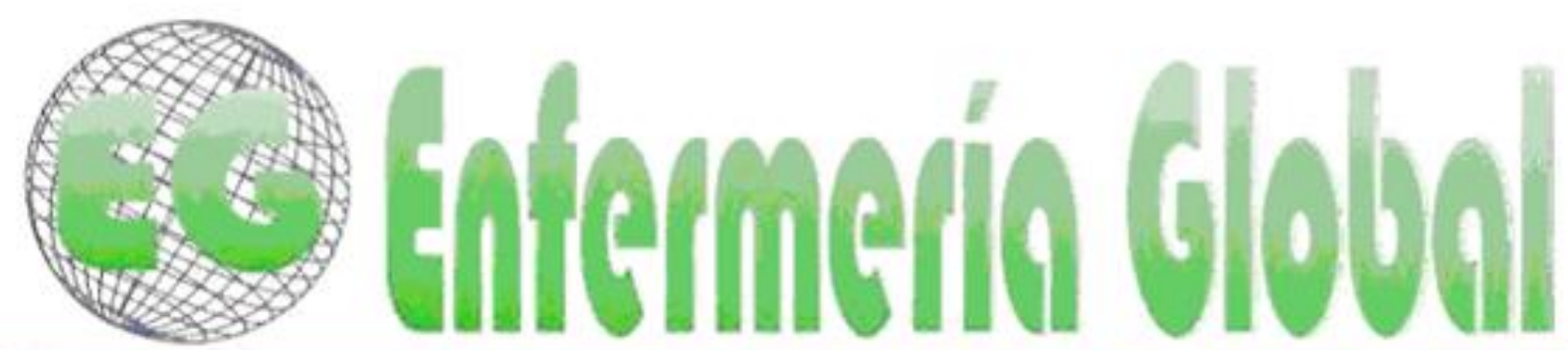

\title{
Relación del Control Glucémico, Función Cognitiva y las Funciones Ejecutivas en el Adulto Mayor con Diabetes Tipo 2: Revisión Sistemática
}

Relation of the Glycemic Control, Cognitive Function and Executive Function in the Elderly with Type 2 Diabetes: Systematic Review

\section{${ }^{*}$ Miranda-Félix, Patricia Enedina *Valles-Ortiz, Patricia Magdalena *Ortiz- Félix, Rosario Edith}

*Estudiante de Doctorado de la Facultad de Enfermería de la Universidad Autónoma de Nuevo León, México E-mail: dulcy84@hotmail.com

\author{
Palabras clave: Función ejecutiva; diabetes mellitus tipo 2; Envejecimiento; Control glucémico; \\ memoria; cognición \\ Keywords: Executive function; type 2 diabetes mellitus; aging; glycemic control; memory; cognition.
}

\section{RESUMEN}

Objetivo: Revisar la evidencia disponible que demuestre la relación que existe entre el control glucémico, función cognitiva y las funciones ejecutivas en el AM con DT2.

Métodos: La búsqueda de la literatura se realizó en idioma inglés y español, en 14 bases de datos, Open acces, y en el buscador Google. En base al modelo propuesto por Cooper (2007), para la síntesis de la literatura. Los estudios fueron evaluados para su validez, a través de la guía CASPe para estudios de casos y controles.

Resultados: Se analizaron 11estudios de correlación, el 100\% de los estudios mostró relación del control glucémico con el deterioro cognitivo y la función ejecutiva en AM con DT2. Los estudios concuerdan que los AM con DT2 presentan deterioro cognitivo, comparado con los AM sin DT2, por lo tanto existe déficit en el $\mathrm{AM}$ al realizar las funciones ejecutivas.

Conclusiones: Según la evidencia disponible existe una relación significativa en el control glucémico y el deterioro cognitivo en el AM con DT2, así mismo un menor desempeño en la movilidad funcional y la fluidez verbal.

\section{ABSTRACT}

Aim: To review the available evidence to show the relationship between glycemic control, cognitive function and executive functions in the AM with T2D. 
Methods: The literature search was conducted in English and Spanish, in 14 databases, Open Access, and the Google search engine. Based on the model proposed by Cooper (2007), for the synthesis of the literature. The studies were assessed for validity through CASPe guide for case-control studies.

Results: We analyzed correlation 11 estudies $100 \%$ of the studies showed glycemic control relationship to cognitive impairment and executive function in AM with DT2. Studies agree that the AM with DT2 have cognitive impairment, compared with AM without DT2, therefore there is a deficit in the AM to perform executive functions.

Conclusions: Based on the available evidence there is a significant relationship in glycemic control and cognitive impairment in the AM with DT2, also a lower performance in functional mobility and verbal fluency.

\section{INTRODUCCIÓN}

La diabetes Tipo 2 (DT2) se ha convertido en un problema de salud pública para el Adulto Mayor (AM), por su alta prevalencia, es una de las enfermedades más común en este grupo de edad. En México, afecta a un 19.2\% de los adultos de 60-69 años, su prevalencia aumenta con la edad a los 75 años, aproximadamente el $20 \%$ de la población la padece y el $44 \%$ tienen más de 65 años ${ }^{(1)}$. La DT2 es un síndrome de etiología múltiple, causada por la falta de insulina y / o la incapacidad de la hormona para ejercer sus efectos adecuadamente, lo que conduce al desarrollo de enfermedades asociadas y complicaciones tales como retinopatía, nefropatía, neuropatía, pérdida de la movilidad articular muscular y fuerza. Además, la función cognitiva también parece estar comprometido en el AM con DT2 ${ }^{(2)}$.

La evidencia muestra que el AM con DT2, tiene mayor probabilidad de presentar deterioro cognitivo debido a los cambios estructurales y funcionales en el Sistema Nervioso Central (SNC), que resulta del proceso de envejecimiento, las estructuras corticales y subcorticales pueden sufrir cambios adicionales debido a cambios en el metabolismo relacionado con la DT2 ${ }^{(3,4)}$

Se ha reportado que el deterioro cognitivo está inversamente relacionado con los niveles del Factor de Crecimiento Insulínico tipo 1(IGF-1) en el AM, un péptido endógeno con múltiples efectos sobre las células neuronales con respuestas neurotróficas, participa en numerosos procesos que mantienen la homeostasis cerebral, por lo que su presencia a concentraciones apropiadas influye en el funcionamiento cerebral ${ }^{(5,6)}$. Existe evidencia de su participación en el mantenimiento neuronal, metabolismo energético, neurogénesis y regulación de los neurotransmisores; asimismo interviene en los procesos de funcionamiento neuronal como la memoria a largo plazo y otras actividades cognitivas ${ }^{(7,8)}$. El deterioro cognitivo probablemente limita los procesos más complejos, relacionados directamente con el lóbulo frontal, como la función ejecutiva, la cual implica un conjunto de habilidades cognitivas, la atención, memoria de trabajo, monitorización, flexibilidad, inhibición, organización, la toma de decisión y conocimiento, que controla la ejecución de actividades complejas, en el contexto de la DT2 esto puede influir para la resolución de problemas en el manejo diario de su condición, memoria de trabajo, y planeación. ${ }^{(3,9)}$. Así mismo el deterioro cognitivo potencialmente puede presentar nuevos obstáculos para el cuidado personal y para lograr el control glucémico $(10,11,12,13)$

En muchas actividades de la vida diaria, las personas necesitan realizar más de una tarea al mismo tiempo, como caminar, comunicarse con otras personas. El deterioro cognitivo durante la ejecución de una tarea doble puede ser un indicador importante 
del estado funcional del AM con DT2. Cuando dos tareas se ejecutan simultáneamente, el rendimiento de uno o ambos puede ser disminuido. La tarea doble, a menudo involucra tanto la información verbal y visual espacial, a medida que el desempeño de tareas simultáneas es complejo, esto podría conducir a un déficit en el manejo del control glucémico ${ }^{(14,15)}$.

Por lo anterior mencionado, en la presente revisión se abordará el control glucémico, función cognitiva y las funciones ejecutivas, con el propósito de revisar la evidencia disponible que demuestre la relación que existe entre el control glucémico, función cognitiva y las funciones ejecutivas en el AM con DT2.

\section{MÉTODOS}

Se realizó una revisión de literatura basándose en los siete pasos de Cooper (16): 1) formulación del problema, 2) búsqueda de literatura, 3) reunir la información de los estudios, 4) evaluación de la calidad de los estudios, 5) analizar e integrar los resultados, 6) interpretar la evidencia y 7) presentar los resultados.

Primeramente se delimitaron las variables a estudiar que fueron: función ejecutiva, función cognitiva, control glucémico. Posteriormente se realizó la búsqueda para identificar revisiones sistemáticas o informes de evaluación en la Biblioteca Cochrane, además de las siguientes bases de datos: Centre for Reviews and Dissemination, Anual review, Dialnet, DynaMed, Medic Latina, Medline Ovid, MEDLINE with Full Text, CINAHL with full text, Embase, Psyc artcicles, Elsevier, Global issues incontext, Academic search complete, Evidence-based Practice Centers, Medline Plus, PubMed, The Campbell Collaboration, Open acces, y en el buscador Google.

Se utilizaron combinaciones de palabras claves de acuerdo al Medical Suject Headings (MeSH) executive function, diabetes, elderly, Blood Glucose SelfMonitoring, attention, memory, cognition en inglés y español. También se realizó una búsqueda manual en las listas de referencias de los artículos de revisión y los estudios incluidos en busca de otros estudios potencialmente elegibles, de octubre a noviembre del 2012, además se utilizaron aperadores boléanos (AND, OR Y NOT) para las bases MEDILE Y EMBASE, se utilizaron truncadores y filtros (\$,”, *, \#) de acuerdo a la estrategia de búsqueda del capítulo 6.4 de Cochrane ${ }^{(17)}$.

Se incluyeron estudios transversales que mostraron relación del control glucémico con las funciones ejecutivas en participantes > de 65 años de edad con DT2, y se excluyeron los estudios con participantes que presentaran algún diagnóstico relacionado con demencia. Para la selección de los artículos primeramente se revisó el resumen del artículo, para identificar los estudios que cumplían con los criterios establecidos. Posteriormente se realizó la lectura en texto completo de los artículos seleccionados, se evaluó la calidad y los riesgos de sesgo a través de su validez interna, se tomó en cuenta el tipo de diseño, tipo de muestreo, tamaño de la muestra, la confiabilidad de los instrumentos, la congruencia del objetivo con los estadísticos utilizados y resultados, también se utilizó la Guía CASPe ${ }^{(18)}$ para evaluar un estudio de casos y controles, la cual consta de 11 preguntas, las primeras dos son de eliminación, si la respuesta a esas dos preguntas fue afirmativa se continuó con las preguntas restantes, de lo contrario se eliminó el estudio, las opciones de respuesta son: sí, no, no sé. La pregunta número 3 de la guía CASpe ${ }^{(18)}$ se eliminó ya que en los estudios evaluados no se dispone de información suficiente para ser respondida, y además no aporta información adicional sobre la calidad de los estudios evaluados, 
en esta etapa se eliminaron algunos estudios por falta de calidad metodológica, ya que mostraban poca congruencia los resultados con los objetivos propuestos. El siguiente diagrama presenta el proceso de selección de los estudios durante las fases de la revisión ${ }^{(19)}$.

Figura 1. Diagrama de flujo de la información a través de las diferentes fases de la revisión sistemática.

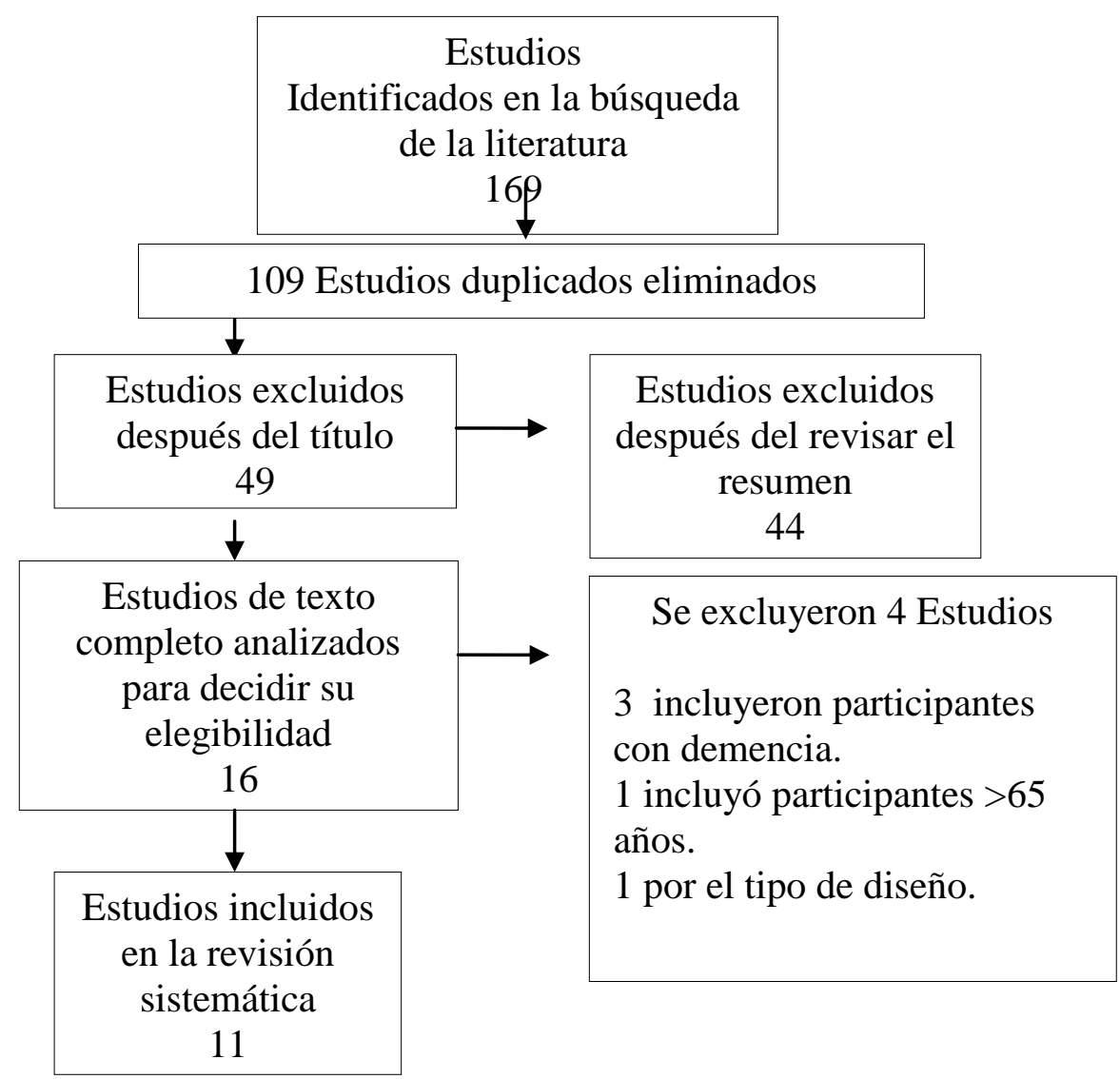

\section{RESULTADOS}

En total se analizaron 11 estudios ${ }^{(2,20-29)}$ que cumplieron con los criterios de inclusión para esta revisión las características se presentan en la Tabla 1. El número de participantes con DT2 incluidos en los estudios varió desde 1398 a 40 participantes por estudio.

Los resultados de esta revisión sistemática proporcionan evidencia de estudios transversales, longitudinales y de casos y control que muestran relación del control glucémico, función cognitiva y las funciones ejecutivas en AM con DT2. Por otra parte estos resultados revelan el impacto que tiene el deterioro cognitivo en el desarrollo de las funciones ejecutivas del AM con DT2. Los estudios analizados demostraron que los niveles de glucosa ocasionan un deterioro cognitivo. Además el deterioro cognitivo se asocia con un déficit en las actividades de cuidado lo cual repercute en el control glucémico ${ }^{(21)}$. Sin embargo, otros estudios mostraron que la capacidad cognitiva afecta la capacidad de los individuos para llevar a cabo el control glucémico, y el control glucémico deficiente, a su vez deteriora la función cognitiva, especialmente en la dieta y el ejercicio ${ }^{(21,22,24,28,29)}$. 
Así mismo se encontró que los niveles de glucosa se asocian con la función cognitiva y un deterioro en la función ejecutiva, por el contrario, en personas sin DT2, no hay una clara asociación entre los niveles basales de glucosa en ayunas, la función ejecutiva y la memoria, ya que no existía relación en los niveles elevados de glucosa $(2,22,23,28)$. De igual manera la DT2 se asocia con el rendimiento en el recuerdo tardío pero no con el recuerdo inmediato. La DT2 se asoció con el deterioro cognitivo y prueba de fluidez verbal, encontraron que el control glucémico deficiente se asocia a alteraciones en el rendimiento en medidas compuestas (tareas dobles) y actividades de la vida diaria de la función ejecutiva ${ }^{(2,22,29)}$. La tabla 1 muestra la relación de las variables de los estudios analizados en esta revisión.

Tabla 1. Artículos Relación de Deterioro Cognitivo y Función Ejecutiva con Control Glucémico

\begin{tabular}{|c|c|c|c|c|c|c|}
\hline \multirow[b]{2}{*}{ Autor/año } & \multirow[b]{2}{*}{$\begin{array}{l}\text { Muestra/ } \\
\text { Diseño }\end{array}$} & \multirow[b]{2}{*}{$\begin{array}{c}\text { Control } \\
\text { glucémico }\end{array}$} & \multirow[b]{2}{*}{$\begin{array}{l}\text { Deterioro } \\
\text { Cognitivo }\end{array}$} & \multicolumn{3}{|c|}{ Executive Functions } \\
\hline & & & & $\begin{array}{l}\text { DLA } \\
(\mathrm{AC})\end{array}$ & $\begin{array}{l}\text { Fluidez } \\
\text { verbal }\end{array}$ & $\begin{array}{l}\text { Tarea } \\
\text { doble }\end{array}$ \\
\hline $\begin{array}{l}\text { Feil, Zhu \& Sultzer, } \\
2012^{(20)}\end{array}$ & $\begin{array}{l}\mathrm{N}=1398 \\
\text { Edad = } 70 \\
\text { Correlacional }\end{array}$ & $\begin{array}{l}\text { Hemoglobina } \\
\text { glucosilada }\end{array}$ & * & * & & \\
\hline $\begin{array}{l}\text { Munshi, Hayes, } \\
\text { Iwata, Lee, } \\
\text { Weinger, } 2012\end{array}$ & $\begin{array}{l}\mathrm{N}=145 \\
\text { Edad } \mathrm{M}=77 \\
\text { correlacional }\end{array}$ & $\begin{array}{l}\text { Hemoglobina } \\
\text { glucosilada }\end{array}$ & * & * & * & \\
\hline $\begin{array}{l}\text { Anagawa et al., } \\
2011^{(22)}\end{array}$ & $\begin{array}{l}\mathrm{N}=16 \\
\text { Edad } \mathrm{M}=70 \\
\text { correlacional }\end{array}$ & $\begin{array}{l}\text { Niveles de } \\
\text { glucosa en } \\
\text { ayunas }\end{array}$ & * & & * & \\
\hline 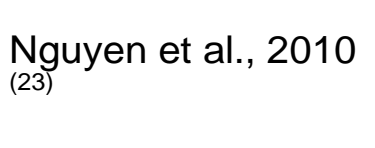 & $\begin{array}{l}\mathrm{N}=95 \\
\text { Edad > } 60 \\
\text { correlacional }\end{array}$ & $\begin{array}{l}\text { Hemoglobina } \\
\text { glucosilada }\end{array}$ & * & * & & \\
\hline 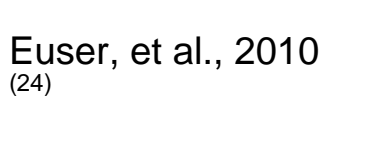 & $\begin{array}{l}\mathrm{N}=3428 \\
\text { Edad=61-97 } \\
\text { correlacional }\end{array}$ & $\begin{array}{l}\text { Niveles de } \\
\text { glucosa en } \\
\text { ayunas }\end{array}$ & * & & & \\
\hline $\begin{array}{l}\text { Van Harten et al } \\
\text { Munshi et al., } \\
2006^{(25)}\end{array}$ & $\begin{array}{l}\mathrm{N}=60 \\
\text { Edad = } 70 \\
\text { correlacional }\end{array}$ & $\begin{array}{l}\text { Hemoglobina } \\
\text { glucosilada }\end{array}$ & * & & & \\
\hline $\begin{array}{l}\text { Van Harten et al., } \\
2006^{(26)}\end{array}$ & $\begin{array}{l}\mathrm{N}=92 \\
\text { Edad } \mathrm{M}=73.2 \\
\text { correlacional }\end{array}$ & $\begin{array}{l}\text { Hemoglobina } \\
\text { glucosilada }\end{array}$ & * & & & \\
\hline $\begin{array}{l}\text { Hagemann, Sartory, } \\
\text { Hader, Kobberli } \\
2005^{(27)}\end{array}$ & $\begin{array}{l}\mathrm{N}=260 ; 129 \mathrm{CD} \\
\text { Edad } 650 \\
\text { Correlacional }\end{array}$ & $\begin{array}{l}\text { Hemoglobina } \\
\text { glucosilada }\end{array}$ & * & * & * & \\
\hline $\begin{array}{l}\text { Alvarenga, Pereirall, } \\
\text { Anjos, } 2005^{(2)}\end{array}$ & $\begin{array}{l}\mathrm{N}=40 ; 20 \mathrm{CD} \\
\text { Edad }=\geq 60 \\
\text { correlacional }\end{array}$ & $\begin{array}{l}\text { Niveles de } \\
\text { glucosa en } \\
\text { ayunas }\end{array}$ & * & & * & * \\
\hline $\begin{array}{l}\text { Debling, Amelang, } \\
\text { Hasselbach, } \\
\text { Sturmer, } 2005^{(28)}\end{array}$ & $\begin{array}{l}\mathrm{N}=740 \\
\text { Edad = 70 } \\
\text { correlacional }\end{array}$ & $\begin{array}{l}\text { Niveles de } \\
\text { glucosa en } \\
\text { ayunas }\end{array}$ & * & * & & \\
\hline $\begin{array}{l}\text { Mogi, et al., } \\
2004^{(29)}\end{array}$ & $\begin{array}{l}\mathrm{N}=69 \\
\text { Edad } 60-85 \\
\text { correlacional }\end{array}$ & $\begin{array}{l}\text { Hemoglobina } \\
\text { glucosilada }\end{array}$ & *- & & & \\
\hline
\end{tabular}

$\mathrm{AVD}=$ Actividades de la Vida Diaria; $\mathrm{AC}=$ Autocuidado; * significancia $\mathrm{p}<0.001$; *significancia negativa. 
Tabla 2. Instrumentos de Medición de las Variables de los Estudios

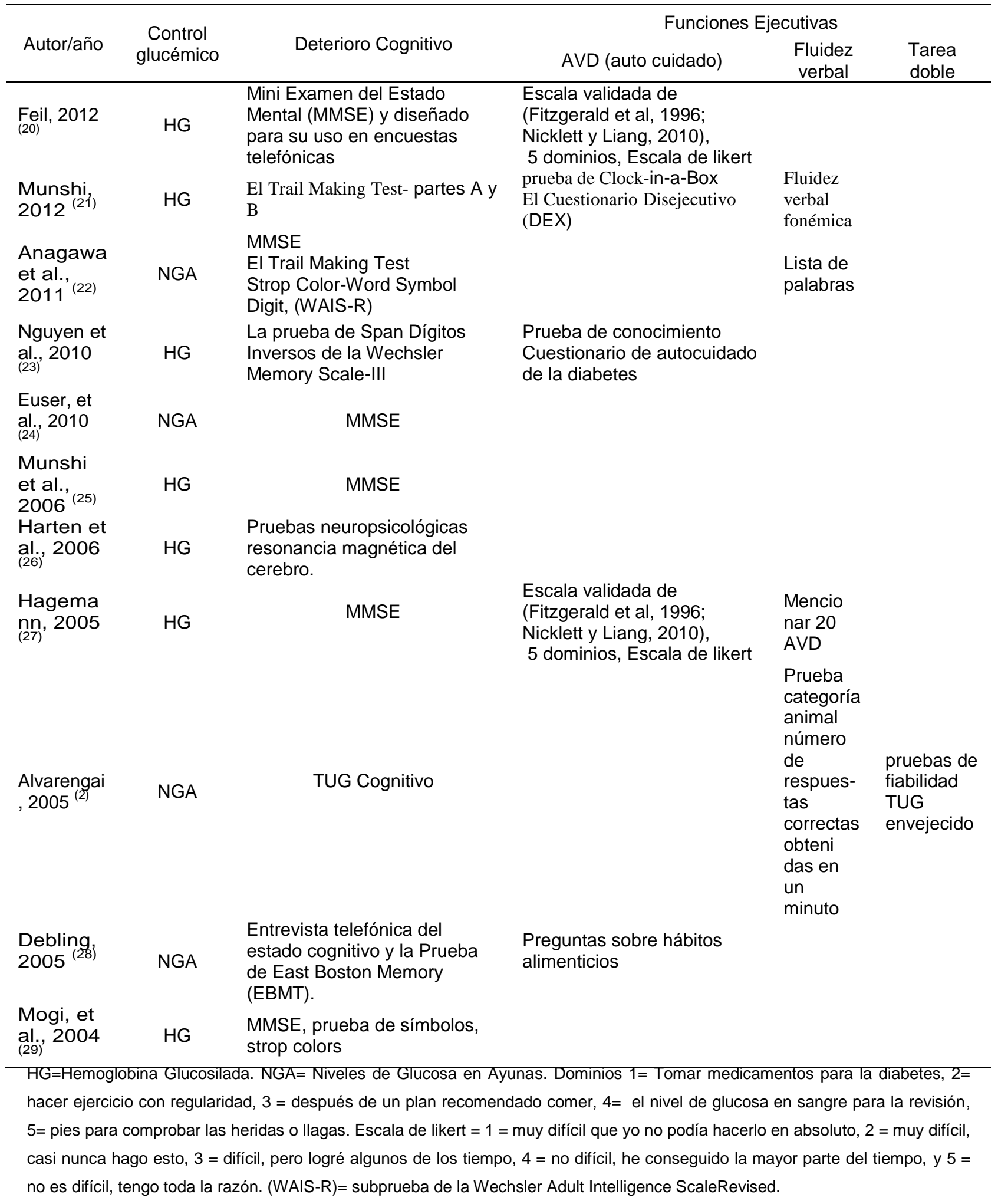

\section{DISCUSIÓN}

El aumento proyectado en la DT2 y el deterioro en la función cognitiva de los adultos mayores presenta un importante problema de salud pública a la sociedad ${ }^{(30,31)}$.

Los resultados obtenidos sugieren una compleja asociación entre la función cognitiva y el control glucémico en esta población. La presente revisión de 11 estudios prueba 
la idea de que la función ejecutiva perjudicará a la DT2 relacionada con el conocimiento y posteriores conductas de auto cuidado, lo que interfiere con el control glucémico. Además el auto cuidado es un componente central de lograr el control glucémico, los datos actuales sugieren que el deterioro de la función ejecutiva puede interferir con la capacidad de los adultos mayores para desempeñar funciones de la vida diaria y relacionada con el cuidado de la DT2. ${ }^{(31)}$

Se ha demostrado que la función ejecutiva puede poner en peligro la capacidad de atención para realizar actividades de la vida diaria, desarrollar tareas complejas necesarias para el auto cuidado, como el control de glucosa en sangre y la insulina, medicación, modificación de la dieta y la actividad física ${ }^{(3,21)}$.

\section{CONCLUSIÓN}

Aunque la función ejecutiva puede estar relacionada con el control glucémico, la comprensión de los mecanismos de tal asociación es limitada. Estos resultados confirman las observaciones anteriores, la presente revisión sugiere seguir indagando los hallazgos que permitan explicar la relación positiva y negativa de la función ejecutiva sobre el control glucémico, se considera necesario realizar investigaciones adicionales para identificar a qué tiempo de ser diagnosticados los AM con DT2, ocurre el deterioro en la función cognitiva, de igual manera realizar intervenciones que permitan examinar con mayor profundidad si la DT2 o la edad la que limita al AM a llevar a cabo las funciones ejecutivas.

\section{REFERENCIAS}

1. Encuesta Nacional de Salud [ENSANUT], (2006). Promoción de Estilos de vida Saludables. Recuperado en http://www.insp.mx/ensanut/ensanut2006.pdf

2. Alvarenga $P$, Pereira $D$, Anjos D. Mobilidade funcional e função executiva em idosos diabéticos e não diabéticos. / Functional mobility and executive function in elderly diabetics and non-diabetics. Brazilian Journal Of Physical Therapy / Revista Brasileira De Fisioterapia [serial on the Internet]. (2010, Nov), [cited November 18, 2012]; 14(6): 491-496.

3. Papazian, O., Alfonso, I. \& Luzondo, R. J. Trastornos de las funciones ejecutivas. Simposio de trastornos del desarrollo. Revista de Neurología, (2006), [cited Noviembre 28, 2012] (2006). 42(3), 45-50.

4. Unger JW, Livingston JN, Moss AM.Insulin receptors in the central nervous system: localization, signalling mechanisms and functional aspects. Prog Neurobiol 1991;36:343-362

5. Isaksson $\mathrm{O}$, Ohlsson $\mathrm{C}$, Nilsson $\mathrm{A}$, et al.Regulation of cartilage growth by growth hormone and insulin-like growth factor I. Pediatr. Nephrol. 1991; 5: 451-453.

6. Trejo J, Carro E, Lopez-Lopez C, Torres-Aleman I.Role of serum insulin-like growth factor I in mammalian brain aging, Growth Hormone \& IGF Res 2004;14 (Suppl. A):S39-S43.

7. Craft S, Watson GS.Insulin and neurodegenerative disease: shared and specific mechanisms. Lancet Neurol 2004;3:169-178.

8. Carro E, Trejo JL, Gerber A, et al.Therapeutic actions of insulin-like growth factor I on APP/PS2 mice with severe brain amyloidosis. Neurobiol Aging 2006; 27:1250-1257.

9. Abbatecola AM, Paolisso G, Lamponi M, et al.Insulin resistance and executive dysfunction in older persons. Journal of the American Geriatrics Society 2004; 52:1713-1718. 
10. Heisler, M., Vijan, S., Anderson, R. M., Ubel, P. A., Bernstein, S. J., \& Hofer, T. P. (2003). When do patients and their physicians agree on diabetes treatment goals and strategies, and what difference does it make? Journal of General Internal Medicine, 18, 893-902.

11. Krapek, K., King, K., Warren, S. S., George, K. G., Caputo, D. A., Mihelich, K., et al. (2004). Medication adherence and associated hemoglobin A1c in type 2 diabetes. Annals of Pharmacotherapy, 38, 1357-1362.

12. Pladevall, M., Williams, L. K., Potts, L. A., Divine, G., Xi, H., \& Lafata, J. E. (2004). Clinical outcomes and adherence to medications measured by claims data in patients with diabetes. Diabetes Care, 27, 2800-2805.

13. Skaer, T. L., Sclar, D. A., Markowski, D. J., \& Won, J. K. (1993). Effect of valueadded utilities on prescription refill compliance and Medicaid health care expenditures-a study of patients with non-insulin-dependent diabetes mellitus. Journal of Clinical Pharmacy Therapeutics, 18, 295-299.

14. Thabit H, Kennelly S, Bhagarva A, Ogunlewe M, McCormack P, Sreenan S, et al. Utilization of Frontal Assessment Battery and Executive Interview 25 in assessing for dysexecutive syndrome and its association with diabetes self-care in elderly patients with type 2 diabetes mellitus. Diabetes Research \& Clinical Practice [serial on the Internet]. (2009, Dec), [cited November 18, 2012]; 86(3): 208-212.

15. Biessels GJ, ter Braak E, Erkelens D, Hijman R.Cognitive function in patients with type 2 diabetes mellitus. Neurosci Res Commun 2001;28:11-22.

16. Cooper H. Research Synthesis and Meta-Analysis. A Step- by -Step Approach. SAGE. $4^{\text {th }}$ ed. Loa Angeles (2009). [Cited Noviembre 2012]; 2, 115-144.

17. Higgins JPT, Green S (editors). Cochrane Handbook for Systematic Reviews of Interventions Version 5.1.0. The Cochrane Collaboration, 2011. [cited November 18, 2012]. Available from. www.cochrane-handbook.org.

18. Guías CASPe de Lectura Crítica de la Literatura Médica. CASPe, D.L.: A-5162005, editors. Cuaderno I. 2008.

19. Urrútia G, Bonfill X. [PRISMA declaration: a proposal to improve the publication of systematic reviews and meta-analyses]. Medicina Clínica [serial on the Internet]. (2010, Oct 9), [cited November 19, 2012]; 135(11): 507-511.

20. Feil D, Zhu C, Sultzer D. The relationship between cognitive impairment and diabetes self-management in a population-based community sample of older adults with Type 2 diabetes. Journal Of Behavioral Medicine [serial on the Internet]. (2012, Apr), [cited November 18, 2012]; 35(2): 190-199.

21. Munshi M, Hayes M, Iwata I, Lee Y, Weinger K. Which aspects of executive dysfunction influence ability to manage diabetes in older adults? Diabetic Medicine [serial on the Internet]. (2012, Sep), [cited November 18, 2012]; 29(9): 1171-1177.

22. Anagawa M, Umegaki H, Uno T, Oyun K, Kawano N, Sato Y, et al. Association between improvements in insulin resistance and changes in cognitive function in elderly diabetic patients with normal cognitive function. Geriatrics \& Gerontology International [serial on the Internet]. (2011, July), [cited November 18, 2012]; 11(3): 341-347.

23. Nguyen H, Grzywacz J, Arcury T, Chapman C, Kirk J, Quandt S, et al. Linking glycemic control and executive function in rural older adults with diabetes mellitus. Journal Of The American Geriatrics Society [serial on the Internet]. (2010, June), [cited November 18, 2012]; 58(6): 1123-1127.

24. Euser S, Sattar N, Witteman J, Bollen E, Sijbrands E, Westendorp R, et al. A prospective analysis of elevated fasting glucose levels and cognitive function in older people: results from PROSPER and the Rotterdam Study. Diabetes [serial on the Internet]. (2010, July), [cited November 18, 2012]; 59(7): 1601-1607. 
25. Munshi M, Grande L, Hayes M, Ayres D, Suhl E, Weinger K, et al. Cognitive dysfunction is associated with poor diabetes control in older adults. Diabetes Care [serial on the Internet]. (2006, Aug), [cited November 19, 2012]; 29(8): 1794-1799.

26. Van Harten B, Oosterman J, Muslimmovic D, Van Loon B, Scheltens P, Weinstein $\mathrm{H}$. Cognitive impairment and MRI correlates in the elderly patients with type 2 diabetes mellitus. Age \& Ageing [serial on the Internet]. (2007, Mar), [cited November 18, 2012]; 36(2): 164-170.

27. Hagemann R, Sartory G, Hader C, Kobberling J. Mood and cognitive function in elderly diabetic patients living in care facilities. Dementia And Geriatric Cognitive Disorders [serial on the Internet]. (2005), [cited November 18, 2012]; 19(5-6): 369-375. 28. Debling D, Amelang M, Hasselbach $\mathrm{P}$, Stürmer T. Diabetes and cognitive function in a population-based study of elderly women and men. Journal Of Diabetes And Its Complications [serial on the Internet]. (2006, July), [cited November 18, 2012]; 20(4): 238-245.

29. Mogi N, Umegaki $\mathrm{H}$, Hattori $\mathrm{A}$, Maeda $\mathrm{N}$, Miura $\mathrm{H}$, Iguchi $\mathrm{A}$, et al. Cognitive function in Japanese elderly with type 2 diabetes mellitus. Journal Of Diabetes And Its Complications [serial on the Internet]. (2004, Jan), [cited November 19, 2012]; 18(1): 42-46.

30. María Fernandes-Lopes R, DE Lima-Argimon I. Idosos com diabetes mellitus tipo 2 e o desempenho cognitivo no teste Wisconsin de classificação de cartas (WCST). Universitas Psychologica [serial on the Internet]. (2010, Sep), [cited November 18, 2012]; 9(3): 697-713.

31. Bell RA, Smith SL, Arcury TA et al. Prevalence and correlates of depressive symptoms among rural older African Americans, Native Americans, and whites with diabetes. Diabetes Care 2005; 28:823-829.

32. Glasgow RE, Fisher L, Skaff $M$ et al. Problem solving and diabetes selfmanagement Investigation in a large, multiracial sample. Diabetes Care 2007; 30:33-37.

Recibido: 23 de mayo 2015; Aceptado: 22 de julio 2015

ISSN 1695-6141

๑ COPYRIGHT Servicio de Publicaciones - Universidad de Murcia 